\title{
Integrable semi-discretizations of the reduced Ostrovsky equation
}

\author{
Bao-Feng Feng ${ }^{1}$, Ken-ichi Maruno ${ }^{2}$ and Yasuhiro Ohta ${ }^{3}$ \\ ${ }^{1}$ Department of Mathematics, The University of Texas-Pan American, Edinburg, TX 78541 \\ 2 Department of Applied Mathematics, Waseda University, Tokyo 169-8050, Japan \\ ${ }^{3}$ Department of Mathematics, Kobe University, Rokko, Kobe 657-8501, Japan \\ E-mail: feng@utpa.edu, kmaruno@waseda.jp and ohta@math.kobe-u.ac.jp
}

\begin{abstract}
Based on our previous work to the reduced Ostrovsky equation (J. Phys. A 45 355203), we construct its integrable semi-discretizations. Since the reduced Ostrovsky equation admits two alternative representations, one is its original form, the other is the differentiation form, or the short wave limit of the Degasperis-Procesi equation, two semidiscrete analogues of the reduced Ostrovsky equation are constructed possessing the same $\mathrm{N}$-loop soliton solution. The relationship between these two versions of semi-discretizations is also clarified.
\end{abstract}

4 July 2018

PACS numbers: 02.30.Ik, 05.45.Yv,42.65.Tg, 42.81.Dp

Accepted by: J. Phys. A: Math. Gen.

\section{Introduction}

In this paper, we consider integrable discretizations of the reduced Ostrovsky equation

$$
\partial_{x}\left(\partial_{t}+u \partial_{x}\right) u-3 u=0
$$

which is a special case $(\beta=0)$ of the Ostrovsky equation

$$
\partial_{x}\left(\partial_{t}+u \partial_{x}+\beta \partial_{x}^{3}\right) u-\gamma u=0 .
$$

The Ostrovsky equation was originally derived as a model for weakly nonlinear surface and internal waves in a rotating ocean [1, 2]. Later on, the same equation was derived for different physical situations by several authors [4, 5]. Especially eq. (1.1) appears as a model for highfrequency waves in a relaxing medium [5, 6]. Note that the reduced Ostrovsky equation (1.1) is sometimes called the Vakhnenko equation [7, 8, 9], the Ostrovsky-Hunter equation [10], or the Ostrovsky-Vakhnenko equation [11, 12]. Travelling wave solutions were investigated in [2, 13, 3]. Vakhnenko et al. constructed the $N$ (loop) soliton solution of the reduced Ostrovsky equation by using a hodograph (reciprocal) transformation and the Hirota bilinear 
method [7, 8]. The same problem was approached from the point of view of inverse scattering method [9].

Differentiating the reduced Ostrovsky equation (1.1), we obtain

$$
u_{t x x}+3 u_{x} u_{x x}+u u_{x x x}-3 u_{x}=0,
$$

which is known as the short wave limit of the Degasperis-Procesi (DP) equation [14, 15]. This equation is derived from the DP equation [16]

$$
U_{T}+3 \kappa^{3} U_{x}-U_{T X X}+4 U U_{X}=3 U_{X} U_{X X}+U U_{X X X},
$$

by taking a short wave limit $\varepsilon \rightarrow 0$ with $U=\varepsilon^{2}\left(u+\varepsilon u_{1}+\cdots\right), T=\varepsilon t, X=\varepsilon^{-1} x$ and $\kappa=1$. It is noted that the short wave limit of the DP equation can also be rewritten as alternative form

$$
\left(\partial_{t}+u \partial_{x}\right) m=-3 m u_{x}, \quad m=1-u_{x x} .
$$

Based on this connection, Matsuno [15] constructed $N$-soliton solution of the short wave model of the DP equation from $N$-soliton solution of the DP equation [17, 18]. This $N$-soliton formula is equivalent to the one obtained by Vakhnenko et al. [7, 8].

As already mentioned previously, the reduced Ostrovsky equation (1.1), as well as its differentiation form (1.3), has attracted much attention in the past. Hone and Wang constructed the Lax pairs for both of equations [14]. The bi-Hamiltonian structure for the reduced Ostrovsky equation (1.1) was found by Brunelli and Sakovich [11], its integrability and wave-breaking was studied in [19]. Interestingly, the short wave limit of the DP equation (1.3) also serves as an asymptotic model for propagation of surface waves in deep water under the condition of small-aspect-ratio [20]. Most recently, the inverse scattering transform (IST) problem for the short wave limit of the DP equation (1.3) was solved by a Riemann-Hilbert approach [12].

The reduced Ostrovsky equation (1.1) is known to be related to the Tzitzeica equation [21, 21, 23, 24], and also the so-called Dodd-Bullough-Mikhailov equation [25, 26, 27], by a reciprocal transformation. Based on this reciprocal link between the reduced Ostrovsky equation and 3-reduction of the B-type or C-type two-dimensional Toda lattice, i.e. the $A_{2}^{(2)}$ 2D-Toda lattice, multi-soliton solutions to both the reduced Ostrovsky equation (1.1) and its differentiation version were constructed by the authors [28].

How to construct its integrable discrete analogue for a soliton equation has been an important topic since the discovery of soliton theory. Although several approaches have been developed starting from the mid-1970s, it remains a challenging and mysterious problem and has to be dealt with on a case by case base. Ablowitz and Ladik originated a method of integrable discretization based the Lax pair of a soliton equation [29, 30]. Almost at the same period, Hirota proposed an intriguing and universal approach based on the bilinear form of a soliton equation [31, 32, 33]. Another successful way to discretize soliton equations was proposed by Date, Jimbo and Miwa [34, 35, 36, 37, 38, 39] via the transformation group theory, which gives a large number of integrable disretizations. One of the most interesting example is the discrete KP equation, or the so-called Hirota-Miwa equation [40, 39], which can be viewed as the Master equation of discrete systems due to the reason that integrable 
discretization of many soliton equations such as discrete $\mathrm{KdV}$ equation, and discrete sineGordon equation can be obtained from Hirota-Miwa equation by reductions. Suris also developed a general Hamiltonian approach for integrable discretizations of integrable systems, see Ref. [41].

The aim of this work is to construct integrable semi-discretizations of the reduced Ostrovsky equation (1.1) and its differentiation form (1.3) by virtue of Hirota's bilinear method. The remainder of the present paper is organized as follows. In section 2, by constructing a semi-discrete analogue of a set of bilinear equations reduced from the period 3-reduction of the $B_{\infty}$ or $C_{\infty}$ two-dimensional Toda system, we derive a semi-discrete reduced Ostrovsky equation based on Eq. (1.3) and provide its $N$-loop soliton solution in terms of pfaffian. Then, an alternative semi-discrete reduced Ostrovsky equation is constructed based on Eq. (1.1) which shares the same $N$-loop soliton solution. It is interesting that a connection between two semi-discrete versions exists in analogue to a link between their continuous counterparts. We conclude our paper by some comments and further topics in section 4 .

\section{Integrable semi-discretization of the short wave limit of the DP equation (1.3)}

It is shown in [28] that bilinear equations for the reduced Ostrovsky equation (1.3) are

$$
\begin{aligned}
& -\left(\frac{1}{2} D_{y} D_{s}-1\right) f \cdot f=f g, \\
& -\left(\frac{1}{2} D_{y} D_{s}-1\right) g \cdot g=f^{2},
\end{aligned}
$$

which originate from a period 3 reduction of BKP (CKP) hierarchy [24]. Here $D_{y} D_{s}$ is the Hirota $D$-operator defined by

$$
D_{y}^{m} D_{s}^{n} f(y, s) \cdot g(y, s)=\left.\left(\frac{\partial}{\partial y}-\frac{\partial}{\partial y^{\prime}}\right)^{m}\left(\frac{\partial}{\partial s}-\frac{\partial}{\partial s^{\prime}}\right)^{n} f(y, s) g\left(y^{\prime}, s^{\prime}\right)\right|_{y=y^{\prime}, s=s^{\prime}}
$$

For the sake of convenience, we set $y=x_{1}, s=x_{-1}$. Under this reduction, one of the taufunctions $f$ turns out to be a square of a pfaffian [42]

$$
\tau^{2}=c f
$$

where $\tau=\operatorname{Pf}(1,2, \cdots, 2 N)$ is a pfaffian whose elements are given by

$$
\operatorname{Pf}(i, j)=c_{i, j}+\frac{p_{i}-p_{j}}{p_{i}+p_{j}} e^{\xi_{i}+\xi_{j}}
$$

with

$$
c_{i, j}=-c_{j, i}, \quad \xi_{i}=p_{i} y+p_{i}^{-1} s+\xi_{i 0}, \quad c=\prod_{i=1}^{2 N} 2 p_{i} .
$$

It was shown in [28] that bilinear equations (2.1)-(2.2), together with (2.3), yield the reduced Ostrovsky equation (1.3) through a hodograph transformation

$$
x=y-2(\ln \tau)_{s}, \quad t=s,
$$


and a dependent transformation

$$
u=-2(\ln \tau)_{s s}=-(\ln f)_{s s} .
$$

Remark 2.1. In accordance with the integrable discretizations which will be constructed hereafter, we choose an alternative hodograph transformation mentioned in Remark 2.15 of [28].

\subsection{Semi-discrete analogues of equations $(2.1)-(2.3)$}

Based on the results briefly mentioned above, we attempt to construct an integrable semidiscrete analogue of the reduced Ostrovsky equation (1.3). The key point is how to discretize the bilinear equations (2.1)-(2.3). To this end, we start with Gram-type determinants

$$
g_{l}=\operatorname{det}_{1 \leq i, j \leq 2 N}\left(m_{i j}(l)\right), \quad f_{l}=\operatorname{det}_{1 \leq i, j \leq 2 N}\left(m_{i j}^{\prime}(l)\right),
$$

where

$$
\begin{gathered}
m_{i j}(l)=C_{i, j}+\frac{1}{p_{i}+p_{j}} \varphi_{i}^{(0)}(l) \varphi_{j}^{(0)}(l), \\
m_{i j}^{\prime}(l)=C_{i, j}+\frac{1}{p_{i}+p_{j}}\left(-\frac{p_{j}}{p_{i}}\right) \frac{1+b p_{i}}{1-b p_{j}} \varphi_{i}^{(0)}(l) \varphi_{j}^{(0)}(l),
\end{gathered}
$$

with

$$
C_{i, j}=C_{j, i}, \quad \varphi_{i}^{(n)}(l)=p_{i}^{n}\left(\frac{1+b p_{i}}{1-b p_{i}}\right)^{l} e^{\xi_{i}}, \quad \xi_{i}=p_{i}^{-1} s+\xi_{i 0} .
$$

Here $2 b$ (not $b)$ is the mesh size in $y$-direction. A relation between $f_{l}$ and $g_{l}$ is shown by the following lemma.

\section{Lemma 2.2.}

$$
\left(D_{s}-2 b\right) g_{l+1} \cdot g_{l}=-2 b f_{l}^{2}
$$

Proof. It can be easily verified that

$$
\begin{aligned}
& \partial_{s} m_{i j}(l)=\varphi_{i}^{(-1)}(l) \varphi_{j}^{(-1)}(l) \\
& m_{i j}(l+1)=m_{i j}(l)+\frac{2 b}{\left(1-b p_{i}\right)\left(1-b p_{j}\right)} \varphi_{i}^{(0)}(l) \varphi_{j}^{(0)}(l),
\end{aligned}
$$

and

$$
m_{i j}^{\prime}(l)=m_{i j}(l)-\frac{1}{1-b p_{j}} \varphi_{i}^{(-1)}(l) \varphi_{j}^{(0)}(l) .
$$

Then by using the following formulas for a $N \times N$ determinant $M$ with $M_{i j}$ denoting the cofactor of the element $m_{i j}$

$$
\frac{\partial}{\partial s}|M|=\sum_{i, j=1}^{N} \frac{\partial m_{i j}}{\partial s} M_{i j}, \quad\left|\begin{array}{cc}
m_{i j} & a_{i} \\
b_{j} & d
\end{array}\right|=d|M|-\sum_{i, j=1}^{N} a_{i} b_{j} M_{i j}
$$


Integrable semi-discretizations of the reduced Ostrovsky equation

we have

$$
\begin{aligned}
& \partial_{s} g_{l}=\left|\begin{array}{cc}
m_{i j}(l) & \varphi_{i}^{(-1)}(l) \\
-\varphi_{j}^{(-1)}(l) & 0
\end{array}\right|, \\
& g_{l+1}=\left|\begin{array}{cc}
m_{i j}(l) & \frac{2 b}{1-b p_{i}} \varphi_{i}^{(0)}(l) \\
-\frac{1}{1-b p_{j}} \varphi_{j}^{(0)}(l) & 1
\end{array}\right|, \\
& f_{l}=\left|\begin{array}{cc}
m_{i j}(l) & \varphi_{i}^{(-1)}(l) \\
\frac{1}{1-b p_{j}} \varphi_{j}^{(0)}(l) & 1
\end{array}\right|=\left|\begin{array}{cc}
m_{i j}(l) & \frac{1}{1-b p_{i}} \varphi_{i}^{(0)}(l) \\
\varphi_{j}^{(-1)}(l) & 1
\end{array}\right| .
\end{aligned}
$$

Furthermore, we can show

$$
\begin{aligned}
& \left(\partial_{s}-2 b\right) g_{l+1}=\left|\begin{array}{ccc}
m_{i j}(l) & \varphi_{i}^{(-1)}(l) & \frac{2 b}{1-b p_{i}} \varphi_{i}^{(0)}(l) \\
-\varphi_{j}^{(-1)}(l) & 0 & 0 \\
-\frac{1}{1-b p_{j}} \varphi_{j}^{(0)}(l) & 0 & 1
\end{array}\right| \\
& +\left|\begin{array}{cc}
m_{i j}(l) & \frac{2 b\left(\partial_{s}-b\right)}{1-b p_{i}} \varphi_{i}^{(0)}(l) \\
-\frac{1}{1-b p_{j}} \varphi_{j}^{(0)}(l) & -b
\end{array}\right|+\left|\begin{array}{cc}
m_{i j}(l) & \frac{2 b}{1-b p_{i}} \varphi_{i}^{(0)}(l) \\
-\frac{\left(\partial_{s}-b\right)}{1-b p_{j}} \varphi_{j}^{(0)}(l) & -b
\end{array}\right| \\
& =\left|\begin{array}{ccc}
m_{i j}(l) & \varphi_{i}^{(-1)}(l) & \frac{2 b}{1-b p_{i}} \varphi_{i}^{(0)}(l) \\
-\varphi_{j}^{(-1)}(l) & 0 & 0 \\
-\frac{1}{1-b p_{j}} \varphi_{j}^{(0)}(l) & 0 & 1
\end{array}\right| \\
& +\left|\begin{array}{cc}
m_{i j}(l) & 2 b \varphi_{i}^{(-1)}(l) \\
-\frac{1}{1-b p_{j}} \varphi_{j}^{(0)}(l) & -b
\end{array}\right|+\left|\begin{array}{cc}
m_{i j}(l) & \frac{2 b}{1-b p_{i}} \varphi_{i}^{(0)}(l) \\
-\varphi_{j}^{(-1)}(l) & -b
\end{array}\right| \\
& =\left|\begin{array}{ccc}
m_{i j}(l) & \varphi_{i}^{(-1)}(l) & \frac{2 b}{1-b p_{i}} \varphi_{i}^{(0)}(l) \\
-\varphi_{j}^{(-1)}(l) & 0 & -2 b \\
-\frac{1}{1-b p_{j}} \varphi_{j}^{(0)}(l) & -1 & 1
\end{array}\right| .
\end{aligned}
$$

By using the Jacobi's identity of determinant and the relations (2.8)-(2.11), we obtain

$$
\left(\partial_{s}-2 b\right) g_{l+1} \times g_{l}=g_{l+1} \times \partial_{s} g_{l}-\left(-2 b f_{l}\right) \times\left(-f_{l}\right),
$$

which is nothing but Eq. (2.7).

Remark 2.3. Eq. (2.7) is an integrable discretization of the bilinear equation (2.3) in $y$ direction. Note that $2 b$ is the mesh size. In the limit of $b \rightarrow 0$, we have

$$
f_{l} \rightarrow f, \quad g_{l} \rightarrow g, \quad g_{l+1} \rightarrow g+2 b g_{y},
$$

then it follows

$$
\frac{1}{2 b} D_{s} g_{l+1} \cdot g_{l} \rightarrow \frac{1}{2} D_{s} D_{y} g \cdot g .
$$


Therefore, equation (2.7) converges to equation (2.3) as $b \rightarrow 0$.

Next, we perform reduction in order to mimic the period 3-reduction of CKP/BKP hierarchy in the continuous case. To this end, we let $C_{i, j}$ take a special value as follows

$$
C_{i, j}=\delta_{j, 2 N+1-i} c_{i}, \quad c_{2 N+1-i}=c_{i},
$$

and further assume

$$
c_{i, j}=-C_{i, j} \frac{2 p_{i}^{2}}{p_{j}} \frac{1-b p_{j}}{1+b p_{i}} .
$$

By imposing a reduction condition

$$
p_{i}^{3}\left(1-b^{2} p_{2 N+1-i}^{2}\right)=-p_{2 N+1-i}^{3}\left(1-b^{2} p_{i}^{2}\right)
$$

which can be written as

$$
\frac{p_{i}^{2}\left(1-b p_{2 N+1-i}\right)}{p_{2 N+1-i}\left(1+b p_{i}\right)}=-\frac{p_{2 N+1-i}^{2}\left(1-b p_{i}\right)}{p_{i}\left(1+b p_{2 N+1-i}\right)},
$$

it then follows

$$
\begin{aligned}
c_{i, j}= & -\delta_{j, 2 N+1-i} c_{i} \frac{2 p_{i}^{2}}{p_{2 N+1-i}} \frac{1-b p_{2 N+1-i}}{1+b p_{i}} \\
& =\delta_{i, 2 N+1-j} c_{2 N+1-i} \frac{2 p_{2 N+1-i}^{2}}{p_{i}} \frac{1-b p_{i}}{1+b p_{2 N+1-i}} \\
& =-c_{j, i} .
\end{aligned}
$$

Thus, we can define a pfaffian

$$
\tau_{l}=\operatorname{Pf}(1,2, \cdots, 2 N)_{l}
$$

whose elements are

$$
(i, j)_{l}=c_{i, j}+\frac{p_{i}-p_{j}}{p_{i}+p_{j}} \varphi_{i}^{(0)}(l) \varphi_{j}^{(0)}(l) .
$$

The relations between the pfaffian $\tau_{l}$ and the Gram-type determinants $f_{l}, g_{l}$ are stated by the following lemma.

\section{Lemma 2.4.}

$$
\begin{aligned}
& \left(D_{s}-b\right) \tau_{l+1} \cdot \tau_{l}=-b c^{\prime} g_{l+1} \\
& \tau_{l}^{2}=c^{\prime} f_{l}
\end{aligned}
$$

where

$$
c^{\prime}=\prod_{i=1}^{2 N} 2 p_{i} \frac{1-b p_{i}}{1+b p_{i}} .
$$

Proof. We firstly list two pfaffian identities which will be used in the process of proof

$$
\operatorname{Pf}_{1 \leq i<j \leq 2 N}\left(\delta \alpha_{i j}-a_{i} b_{j}+a_{j} b_{i}\right)=\operatorname{Pf}\left(\begin{array}{ccc}
\alpha_{i j} & a_{i} & b_{i} \\
& & \delta
\end{array}\right),
$$


Integrable semi-discretizations of the reduced Ostrovsky equation

$$
\begin{gathered}
\underset{1 \leq i, j \leq 2 N}{\operatorname{det}}\left(\begin{array}{ccc}
\alpha_{i j} & a_{i} & b_{i} \\
c_{j} & \alpha & \beta \\
d_{j} & \gamma & \delta
\end{array}\right)=\operatorname{Pf}\left(\alpha \alpha_{i j}-a_{i} c_{j}+a_{j} c_{i}\right) \operatorname{Pf}\left(\delta \alpha_{i j}-b_{i} d_{j}+b_{j} d_{i}\right) \\
-\operatorname{Pf}\left(\gamma \alpha_{i j}-a_{i} d_{j}+a_{j} d_{i}\right) \operatorname{Pf}\left(\beta \alpha_{i j}-b_{i} c_{j}+b_{j} c_{i}\right)
\end{gathered}
$$

Since

$$
\begin{gathered}
\partial_{s}(i, j)_{l}=\varphi_{i}^{(0)}(l) \varphi_{j}^{(-1)}(l)-\varphi_{i}^{(-1)}(l) \varphi_{j}^{(0)}(l), \\
(i, j)_{l+1}=(i, j)_{l}+\varphi_{i}^{(0)}(l+1) \varphi_{j}^{(0)}(l)-\varphi_{i}^{(0)}(l) \varphi_{j}^{(0)}(l+1), \\
\left.\left(\partial_{\tau}-b\right)\right)(i, j)_{l+1}=-b(i, j)_{l}+\varphi_{i}^{(0)}(l+1) \varphi_{j}^{(-1)}(l)-\varphi_{i}^{(-1)}(l) \varphi_{j}^{(0)}(l+1),
\end{gathered}
$$

we have

$$
\begin{aligned}
& \tau_{l}=\operatorname{Pf}\left(\begin{array}{ccc}
(i, j)_{l} & \varphi_{i}^{(0)}(l) & \varphi_{i}^{(0)}(l) \\
& & 1
\end{array}\right) \\
& \partial_{s} \tau_{l}=\operatorname{Pf}\left(\begin{array}{ccc}
(i, j)_{l} & \varphi_{i}^{(-1)}(l) & \varphi_{i}^{(0)}(l) \\
& & 0
\end{array}\right), \\
& \tau_{l+1}=\operatorname{Pf}\left(\begin{array}{ccc}
(i, j)_{l} & \varphi_{i}^{(0)}(l) & \varphi_{i}^{(0)}(l+1) \\
& & 1
\end{array}\right), \\
& \left(\partial_{s}-b\right) \tau_{l+1}=\operatorname{Pf}\left(\begin{array}{ccc}
(i, j)_{l} & \varphi_{i}^{(-1)}(l) & \varphi_{i}^{(0)}(l+1) \\
& & -b
\end{array}\right),
\end{aligned}
$$

by referring to the identity (2.17). Furthermore, by using the pfaffian identify (2.18)

$$
\begin{aligned}
& \left(D_{s}-b\right) \tau_{l+1} \cdot \tau_{l}=\tau_{l}\left(\partial_{s}-b\right) \tau_{l+1}-\tau_{l+1} \partial_{s} \tau_{l} \\
& =\operatorname{Pf}\left(\begin{array}{ccc}
(i, j)_{l} & \varphi_{i}^{(-1)}(l) & \varphi_{i}^{(0)}(l+1) \\
& & -b
\end{array}\right) \operatorname{Pf}\left(\begin{array}{ccc}
(i, j)_{l} & \varphi_{i}^{(0)}(l) & \varphi_{i}^{(0)}(l+1) \\
& & 1
\end{array}\right) \\
& -\operatorname{Pf}\left(\begin{array}{ccc}
(i, j)_{l} & \varphi_{i}^{(0)}(l) & \varphi_{i}^{(0)}(l) \\
& & 1
\end{array}\right) \operatorname{Pf}\left(\begin{array}{ccc}
(i, j)_{l} & \varphi_{i}^{(-1)}(l) & \varphi_{i}^{(0)}(l) \\
& 0
\end{array}\right) \\
& =\left|\begin{array}{ccc}
m_{i j}(l) & \varphi_{i}^{(-1)}(l) & \varphi_{i}^{(0)}(l) \\
\varphi_{j}^{(0)}(l+1) & -b & 1 \\
\varphi_{j}^{(0)}(l) & 0 & 1
\end{array}\right| \\
& =\left|\begin{array}{ccc}
m_{i j}(l)-\varphi_{i}^{(0)}(l) \varphi_{j}^{(0)}(l) & \varphi_{i}^{(-1)}(l) & \varphi_{i}^{(0)}(l) \\
\varphi_{j}^{(0)}(l+1)-\varphi_{j}^{(0)}(l) & -b & 1 \\
0 & 0 & 1
\end{array}\right| \\
& =\left|\begin{array}{cc}
m_{i j}(l)-\varphi_{i}^{(1)}(l) \varphi_{j}^{(1)}(l) & \varphi_{i}^{(-1)}(l) \\
b\left(\varphi_{j}^{(0)}(l+1)+\varphi_{j}^{(0)}(l)\right) & -b
\end{array}\right| \\
& =-b \operatorname{det}\left(m_{i j}(l)-\varphi_{i}^{(0)}(l) \varphi_{j}^{(0)}(l)+\varphi_{i}^{(-1)}(l)\left(\varphi_{j}^{(1)}(l+1)+\varphi_{j}^{(1)}(l)\right)\right)
\end{aligned}
$$


Integrable semi-discretizations of the reduced Ostrovsky equation

$$
\begin{aligned}
& =-b \operatorname{det}\left(c_{i, j}+\frac{2 p_{j}^{2}\left(1+b p_{i}\right)}{p_{i}\left(p_{i}+p_{j}\right)\left(1-b p_{j}\right)} \varphi_{i}^{(0)}(l) \varphi_{j}^{(0)}(l)\right) \\
& =-b c^{\prime} \operatorname{det}\left(\frac{p_{i}\left(1-b p_{j}\right)}{2 p_{j}^{2}\left(1+b p_{i}\right)} c_{i, j}+\frac{1}{p_{i}+p_{j}} \varphi_{i}^{(0)}(l) \varphi_{j}^{(0)}(l)\right) \\
& =-b c^{\prime} \operatorname{det}\left(C_{i, j}+\frac{1}{p_{i}+p_{j}} \varphi_{i}^{(0)}(l) \varphi_{j}^{(0)}(l)\right) \\
& =-b c^{\prime} F_{l+1} .
\end{aligned}
$$

Thus Eq. (2.15) is proved. Next, we prove the relation (2.16).

$$
\begin{aligned}
& f_{l}=\operatorname{det}\left(\delta_{j, 2 N+1-i} c_{i}-\frac{p_{j}}{p_{i}} \frac{1}{p_{i}+p_{j}} \frac{1+b p_{i}}{1-b p_{j}} \varphi_{i}^{(0)}(l) \varphi_{j}^{(0)}(l)\right) \\
& =\prod_{i=1}^{2 N} \frac{1+b p_{i}}{2 p_{i}\left(1-b p_{i}\right)} \operatorname{det}\left(-\delta_{j, 2 N+1-i} c_{i} \frac{2 p_{i}^{2}}{p_{j}} \frac{1-b p_{j}}{1+b p_{i}}+\frac{2 p_{i}}{p_{i}+p_{j}} \varphi_{i}^{(0)}(l) \varphi_{j}^{(0)}(l)\right) \\
& =\frac{1}{c^{\prime}} \operatorname{det}\left(c_{i, j}+\left(\frac{p_{i}-p_{j}}{p_{i}+p_{j}}-1\right) \varphi_{i}^{(0)}(l) \varphi_{j}^{(0)}(l)\right) \\
& =\frac{1}{c^{\prime}}\left[\operatorname{Pf}\left(c_{i, j}+\frac{p_{i}-p_{j}}{p_{i}+p_{j}} \varphi_{i}^{(0)}(l) \varphi_{j}^{(0)}(l)\right)\right]^{2} .
\end{aligned}
$$

Therefore Eq. (2.16) holds.

Remark 2.5. Obviously, Eq. (2.16) converges to (2.3) as $b \rightarrow 0$ since $\tau_{l} \rightarrow \tau, f_{l} \rightarrow f$ and $c^{\prime} \rightarrow c$ under this limit.

Remark 2.6. Multiplying both sides of Eq. (2.15) by $2 \tau_{l} \tau_{l+1}$, we have

$$
\left(D_{s}-2 b\right) \tau_{l+1}^{2} \cdot \tau_{l}^{2}=-2 b c^{\prime} g_{l+1} \tau_{l+1} \tau_{l}
$$

by using a bilinear identify $D_{s} f^{2} \cdot g^{2}=2 f g D_{s} f \cdot g$. Furthermore, by referring to the relation (2.3), we have

$$
\left(\frac{1}{2 b} D_{s}-1\right) f_{l+1}^{2} \cdot f_{l}^{2}=-\frac{1}{c^{\prime}} g_{l+1} \tau_{l+1} \tau_{l}
$$

which converges to (2.1) as $b \rightarrow 0$ since $g_{l+1} \rightarrow g$ and $\tau_{l+1} \tau_{l} / c^{\prime} \rightarrow \tau^{2} / c=f$ under this limit.

\subsection{Integrable semi-discretization of the short wave limit of the DP equation (1.3)}

Summarizing what we have discussed in the previous subsection, the following three relations

$$
\begin{aligned}
& \left(D_{s}-2 b\right) g_{l+1} \cdot g_{l}=-2 b f_{l}^{2}, \\
& \left(D_{s}-b\right) \tau_{l+1} \cdot \tau_{l}=-b c^{\prime} g_{l+1}, \\
& \tau_{l}^{2}=c^{\prime} f_{l} .
\end{aligned}
$$

constitute the semi-discrete analogue of bilinear equations (2.1)-(2.3). Let us construct integrable semi-discretization of the reduced Ostrovsky equation based on bilinear equations (2.25)-(2.27). First, we rewrite Eqs. (2.25) and (2.26) into

$$
\left(\ln \frac{g_{l+1}}{g_{l}}\right)_{s}-2 b=-2 b \frac{f_{l}^{2}}{g_{l+1} g_{l}},
$$


Integrable semi-discretizations of the reduced Ostrovsky equation

and

$$
\left(\ln \frac{\tau_{l+1}}{\tau_{l}}\right)_{s}-b=-b c^{\prime} \frac{g_{l+1}}{\tau_{l+1} \tau_{l}}
$$

respectively. Introducing a discrete hodograph transformation

$$
x_{l}=2 l b-2\left(\ln \tau_{l}\right)_{s}, \quad t=s,
$$

and a dependent variable transformation

$$
u_{l}=-2\left(\ln \tau_{l}\right)_{s s}=-\left(\ln f_{l}\right)_{s s}
$$

it then follows that the nonuniform mesh, which is defined by $\delta_{l}=x_{l+1}-x_{l}$, can be expressed as

$$
\delta_{l}=2 b-2\left(\ln \frac{\tau_{l+1}}{\tau_{l}}\right)_{s}=2 b c^{\prime} \frac{g_{l+1}}{\tau_{l+1} \tau_{l}},
$$

with the use of (2.29). Differentiating Eq. (2.32) with respect to $s$, one obtains

$$
\frac{d \delta_{l}}{d s}=-2\left(\ln \frac{\tau_{l+1}}{\tau_{l}}\right)_{s s}=u_{l+1}-u_{l} .
$$

Introducing an auxiliary variable $r_{l}=f_{l} / g_{l}$, we then have

$$
\frac{4}{\delta_{l}^{2}}=\frac{1}{b^{2}} \frac{g_{l}}{g_{l+1}} r_{l} r_{l+1}
$$

where (2.27) is used. Further, one obtains

$$
\left(\ln \frac{r_{l+1}}{r_{l}}\right)_{s}+\delta_{l}=2 b \frac{f_{l}^{2}}{g_{l+1} g_{l}}
$$

by referring to (2.29) and (2.28). Taking the logarithmic derivative of (2.34) with respect to $s$ leads to

$$
\left(\ln r_{l+1} r_{l}\right)_{s}-\left(\ln \frac{g_{l+1}}{g_{l}}\right)_{s}=-\frac{2}{\delta_{l}} \frac{d \delta_{l}}{d s} .
$$

Substituting Eq.(2.33) into Eq. (2.36) and referring to Eqs. and (2.28) and (2.35), one obtains

$$
\begin{aligned}
\frac{u_{l+1}-u_{l}}{\delta_{l}} & =-\frac{1}{2}\left(\ln r_{l+1} r_{l}\right)_{s}+\frac{1}{2}\left(\ln \frac{g_{l+1}}{g_{l}}\right)_{s} \\
& =-\frac{1}{2}\left(\ln r_{l+1} r_{l}\right)_{s}+b-b \frac{f_{l}^{2}}{g_{l} g_{l+1}} \\
& =-\frac{1}{2}\left(\ln r_{l+1} r_{l}\right)_{s}+b-\frac{1}{2}\left(\ln \frac{r_{l+1}}{r_{l}}\right)_{s}-\frac{1}{2} \delta_{l} \\
& =-\left(\ln r_{l+1}\right)_{s}+b-\frac{1}{2} \delta_{l},
\end{aligned}
$$

which can be recast into

$$
\left(\ln r_{l+1}\right)_{s}=-\frac{u_{l+1}-u_{l}}{\delta_{l}}+b-\frac{1}{2} \delta_{l} .
$$

A substitution of (2.38) back into (2.35) leads to

$$
-\frac{u_{l+1}-u_{l}}{\delta_{l}}+\frac{u_{l}-u_{l-1}}{\delta_{l-1}}+\frac{1}{2} \delta_{l}+\frac{1}{2} \delta_{l-1}=\frac{8 b^{3}}{\delta_{l}^{2}} \frac{r_{l}}{r_{l+1}} .
$$


Integrable semi-discretizations of the reduced Ostrovsky equation

Defining

$$
m_{l}=\frac{2}{\delta_{l}+\delta_{l-1}}\left(-\frac{u_{l+1}-u_{l}}{\delta_{l}}+\frac{u_{l}-u_{l-1}}{\delta_{l-1}}\right)+1,
$$

and taking the logarithmic derivative on both sides of (2.39), we have

$$
\begin{aligned}
& \frac{d \ln m_{l}}{d s}=\left(\ln r_{l}\right)_{s}-\left(\ln r_{l+1}\right)_{s}-\frac{2}{\delta_{l}} \frac{d \delta_{l}}{d s}-\frac{d}{d s}\left(\delta_{l}+\delta_{l-1}\right) \\
= & -\frac{u_{l}-u_{l-1}}{\delta_{l-1}}-\frac{1}{2} \delta_{l-1}+\frac{u_{l+1}-u_{l}}{\delta_{l}}+\frac{1}{2} \delta_{l}-\frac{2\left(u_{l+1}-u_{l}\right)}{\delta_{l}}-\frac{u_{l+1}-u_{l-1}}{\delta_{l}+\delta_{l-1}} \\
= & -\frac{u_{l}-u_{l-1}}{\delta_{l-1}}-\frac{u_{l+1}-u_{l}}{\delta_{l}}-\frac{u_{l+1}-u_{l-1}}{\delta_{l}+\delta_{l-1}}-\frac{1}{2}\left(\delta_{l}-\delta_{l-1}\right) .
\end{aligned}
$$

As a result, by defining forward difference and average operators

$$
\Delta u_{l}=\frac{u_{l+1}-u_{l}}{\delta_{l}}, \quad M u_{l}=\frac{u_{l}+u_{l-1}}{2},
$$

we can summarize what we have deduced into the following theorem.

Theorem 2.7. The semi-discrete analogue of the short wave limit of the DP equation

$$
\left\{\begin{array}{l}
\frac{d m_{l}}{d s}=m_{l}\left(-2 M \Delta u_{l}-\frac{M\left(\delta_{l} \Delta u_{l}\right)}{M \delta_{l}}-\frac{1}{2}\left(\delta_{l}-\delta_{l-1}\right)\right), \\
\frac{d \delta_{l}}{d s}=u_{l+1}-u_{l} \\
m_{l}=-2 \frac{M \Delta u_{l}}{M \delta_{l}}+1,
\end{array}\right.
$$

is determined from the following equations

$$
\left\{\begin{array}{l}
\left(D_{s}-2 b\right) g_{l+1} \cdot g_{l}=-2 b f_{l}^{2}, \\
\left(D_{s}-b\right) f_{l+1} \cdot f_{l}=-b c^{\prime} g_{l+1}, \\
\tau_{l}^{2}=c^{\prime} f_{l},
\end{array}\right.
$$

through discrete hodograph transformation $x_{l}=2 l b-2\left(\ln \tau_{l}\right)_{s}, \delta_{l}=x_{l+1}-x_{l}, t=s$ and dependent variable transformation $u_{l}=-2\left(\ln \tau_{l}\right)_{s s}=-\left(\ln f_{l}\right)_{s s}$.

Let us consider the continuous limit when $b \rightarrow 0$. The dependent variable $u$ is a function of $l$ and $s$. Meanwhile, we regard it as a function of $x$ and $t$, where $x$ is the space coordinate at $l$-th lattice point and $t$ is the time, defined by

$$
x=x_{0}+\sum_{j=0}^{l-1} \delta_{j}, \quad t=s
$$

Then in the continuous limit, $b \rightarrow 0\left(\delta_{l} \rightarrow 0\right)$, we have

$$
\begin{aligned}
2 M \Delta u_{l} & =\frac{u_{l+1}-u_{l}}{\delta_{l}}+\frac{u_{l}-u_{l-1}}{\delta_{l-1}} \rightarrow 2 u_{x}, \quad \frac{M\left(\delta_{l} \Delta u_{l}\right)}{M \delta_{l}}=\frac{u_{l+1}-u_{l-1}}{\delta_{l}+\delta_{l-1}} \rightarrow u_{x}, \\
m_{l} & =\frac{2}{\delta_{l}+\delta_{l-1}}\left(-\frac{u_{l+1}-u_{l}}{\delta_{l}}+\frac{u_{l}-u_{l-1}}{\delta_{l-1}}\right)+1 \rightarrow m=-u_{x x}+1 .
\end{aligned}
$$


Moreover, since

$$
\frac{\partial x}{\partial s}=\frac{\partial x_{0}}{\partial s}+\sum_{j=0}^{l-1} \frac{\partial \delta_{j}}{\partial s}=\frac{\partial x_{0}}{\partial s}+\sum_{j=0}^{l-1}\left(u_{j+1}-u_{j}\right) \rightarrow u
$$

we then have

$$
\partial_{s}=\partial_{t}+\frac{\partial x}{\partial s} \partial_{x} \rightarrow \partial_{t}+u \partial_{x}
$$

Consequently, the third equation in (2.41) converges to $m=1-u_{x x}$. Whereas the first equation in (2.41) converges to

$$
\left(\partial_{t}+u \partial_{x}\right) m=-3 m u_{x}
$$

which is exactly the short wave limit of the DP equation (1.3).

Based on the results in previous section, we can provide $N$-soliton solution to the semidiscrete reduced Ostrovsky equation

Theorem 2.8. The $N$-soliton solution to the semi-discrete analogue for the short wave limit of the DP equation (2.41) takes the following parametric form

$$
u_{l}=-2\left(\ln \tau_{l}\right)_{s s}, \quad x_{l}=2 l b-2\left(\ln \tau_{l}\right)_{s}
$$

where $\tau_{l}$ is a pfaffian

$$
\tau_{l}=\operatorname{Pf}(1,2, \cdots, 2 N)_{l},
$$

whose elements are

$(i, j)_{l}=c_{i, j}+\frac{p_{i}-p_{j}}{p_{i}+p_{j}} \varphi_{i}^{(0)}(l) \varphi_{j}^{(0)}(l), \quad \varphi_{i}^{(n)}(l)=p_{i}^{n}\left(\frac{1+b p_{i}}{1-b p_{i}}\right)^{l} e^{p_{i}^{-1} s+\xi_{i 0}}$

under the reduction condition

$$
p_{i}^{3}\left(1-b^{2} p_{2 N+1-i}^{2}\right)=-p_{2 N+1-i}^{3}\left(1-b^{2} p_{i}^{2}\right), \quad i=1,2, \cdots, N .
$$

\section{Integrable semi-discretization of the reduced Ostrovsky equation (1.1)}

\subsection{Bilinear equation for the reduced Ostrovsky equation (1.1)}

In this section, we will deduce an integrable semi-discrete analogue to the reduced Ostrovsky equation (1.1). It was pointed out by the authors [28] that a single bilinear equation (2.53) yields the reduced Ostrovsky equation (1.1). In order to be consistent with the $N$-soliton solution given in the previous section, we start with

$$
\left[\left(D_{x_{-3}}-D_{x_{-1}}^{3}\right) D_{x_{1}}+3 D_{x_{-1}}^{2}\right] \tau \cdot \tau=0,
$$

which is a dual bilinear equation of (2.47) in [28] for the extended BKP hierarchy. Imposing the same period 3-reduction by requesting $D_{x_{3}}=D_{x_{-3}}=0$ and assuming $y=x_{1}, s=x_{-1}$, Eq. (3.1) is reduced to

$$
\left(D_{y} D_{s}^{3}-3 D_{s}^{2}\right) \tau \cdot \tau=0 .
$$


Prior to proceeding to the semi-discretization of Eq. (1.1), let us briefly show how the reduced Ostrovsky equation (1.1) is derived from Eq. (3.2) through the same hodograph transformation (2.5) and dependent variable transformation (2.6) defined in the previous section. By defining $\rho^{-1}=1-2(\ln \tau)_{y s}$, a conversion formula

$$
\left\{\begin{array}{l}
\frac{\partial}{\partial y}=\frac{1}{\rho} \frac{\partial}{\partial x}, \\
\frac{\partial}{\partial s}=\frac{\partial}{\partial t}+u \frac{\partial}{\partial x},
\end{array}\right.
$$

can be easily obtained from the hodograph transformation (2.5). By using the relations

$$
\begin{aligned}
& \frac{D_{y} D_{s}^{3} \tau \cdot \tau}{\tau^{2}}=2(\ln \tau)_{y s s s}+12(\ln \tau)_{s s}(\ln \tau)_{y s}, \\
& \frac{D_{s}^{2} \tau \cdot \tau}{\tau^{2}}=2(\ln \tau)_{s s},
\end{aligned}
$$

Eq. (3.2) is converted to

$$
2(\ln \tau)_{y s s s}=6(\ln \tau)_{s s}\left(1-2(\ln \tau)_{y s}\right),
$$

and is further reduced to

$$
\rho u_{x_{1} x_{-1}}=3 u \text {. }
$$

With the use of the conversion formulas (3.3), we finally arrive at

$$
\partial_{x}\left(\partial_{t}+u \partial_{x}\right) u=3 u
$$

which is exactly the reduced Ostrovsky equation (1.1).

\subsection{Semi-discrete analogue of the reduced Ostrovsky equation (1.1)}

In order to obtain a discrete analogue for the bilinear equation (3.2), we first prove a bilinear equation associated with the modified BKP.

Lemma 3.1. Assume a pfaffian $\tau_{l}=\operatorname{Pf}(1,2, \cdots, 2 N)_{l}$ with element determined by

$$
(i, j)_{l}=c_{i, j}+\frac{p_{i}-p_{j}}{p_{i}+p_{j}} \varphi_{i}^{(0)}(l) \varphi_{j}^{(0)}(l)
$$

where

$$
\varphi_{i}^{(n)}(l)=p_{i}^{n}\left(\frac{1+b p_{i}}{1-b p_{i}}\right)^{l} e^{\xi_{i}}, \quad \xi_{i}=p_{i}^{-1} s+p_{i}^{-3} r+\xi_{i 0} .
$$

Then the pfaffian $\tau_{l}$ satisfies the following bilinear equation

$$
\left(\left(D_{s}-b\right)^{3}-\left(D_{r}-b^{3}\right)\right) \tau_{l+1} \cdot \tau_{l}=0 .
$$

Proof. First, we define the pfaffian elements in addition to (3.7):

$$
\begin{gathered}
\operatorname{Pf}\left(i, d_{n}\right)_{l}=\varphi_{i}^{(n)}(l), \quad \operatorname{Pf}\left(d_{m}, d_{n}\right)_{l}=0, \\
\operatorname{Pf}\left(i, d^{l}\right)_{l}=\varphi_{i}^{(0)}(l+1), \quad\left(d_{m}, d^{k}\right)_{k l}=(-b)^{-m} .
\end{gathered}
$$


Integrable semi-discretizations of the reduced Ostrovsky equation

Then the following differential and difference formulas are obtained previously or can be easily verified

$$
\begin{aligned}
& \partial_{s} \tau_{l}=\operatorname{Pf}\left(\begin{array}{ccc}
(i, j)_{l} & \varphi_{i}^{(-1)}(l) & \varphi_{i}^{(0)}(l) \\
& 0
\end{array}\right)=\operatorname{Pf}\left(1,2, \cdots, 2 N, d_{-1}, d_{0}\right)_{l}, \\
& \partial_{s}^{2} \tau_{l}=\operatorname{Pf}\left(\begin{array}{ccc}
(i, j)_{l} & \varphi_{i}^{(-2)}(l) & \varphi_{i}^{(0)}(l) \\
& & 0
\end{array}\right)=\operatorname{Pf}\left(1,2, \cdots, 2 N, d_{-2}, d_{0}\right)_{l} \\
& \tau_{l+1}=\operatorname{Pf}\left(\begin{array}{ccc}
(i, j)_{l} & \varphi_{i}^{(0)}(l) & \varphi_{i}^{(0)}(l+1) \\
& & 1
\end{array}\right)=\operatorname{Pf}\left(1,2, \cdots, 2 N, d_{0}, d^{l}\right)_{l}, \\
& \left(\partial_{s}-b\right) \tau_{l+1}=\operatorname{Pf}\left(\begin{array}{ccc}
(i, j)_{l} & \varphi_{i}^{(-1)}(l) & \varphi_{i}^{(0)}(l+1) \\
& & -b
\end{array}\right)=\operatorname{Pf}\left(1,2, \cdots, 2 N, d_{-1}, d^{l}\right)_{l}, \\
& \left(\partial_{s}-b\right)^{2} \tau_{l+1}=\operatorname{Pf}\left(\begin{array}{ccc}
(i, j)_{l} & \varphi_{i}^{(-2)}(l) & \varphi_{i}^{(0)}(l+1) \\
& & b^{2}
\end{array}\right)=\operatorname{Pf}\left(1,2, \cdots, 2 N, d_{-2}, d^{l}\right)_{l}, \\
& \frac{1}{3}\left(\partial_{s}^{3}-\partial_{r}\right) \tau_{l}=\operatorname{Pf}\left(\begin{array}{ccc}
(i, j)_{l} & \varphi_{i}^{(-2)}(l) & \varphi_{i}^{(-1)}(l) \\
& 0
\end{array}\right)=\operatorname{Pf}\left(1,2, \cdots, 2 N, d_{-2}, d_{-1}\right)_{l}
\end{aligned}
$$

Moreover, the following relations can be further verified

$$
\begin{aligned}
& \left(\partial_{r}-b^{3}\right) \tau_{l+1}=\operatorname{Pf}\left(\begin{array}{ccc}
(i, j)_{l} & \varphi_{i}^{(-3)}(l) & \varphi_{i}^{(0)}(l+1) \\
& & -b^{3}
\end{array}\right) \\
& -2 \operatorname{Pf}\left(\begin{array}{ccccc}
(i, j)_{l} & \varphi_{i}^{(-2)}(l) & \varphi_{i}^{(-1)}(l) & \varphi_{i}^{(0)}(l) & \varphi_{i}^{(0)}(l+1) \\
& & 0 & 0 & b^{2} \\
& & & 0 & -b \\
& & & &
\end{array}\right) \\
& =\operatorname{Pf}\left(1,2, \cdots, 2 N, d_{-1}, d^{l}\right)_{l}-2 \operatorname{Pf}\left(1,2, \cdots, 2 N, d_{-2}, d_{-1}, d_{0}, d^{l}\right)_{l} \text {, } \\
& \left(\partial_{s}-b\right)^{3} \tau_{l+1}=\operatorname{Pf}\left(\begin{array}{ccc}
(i, j)_{l} & \varphi_{i}^{(-3)}(l) & \varphi_{i}^{(0)}(l+1) \\
& & -b^{3}
\end{array}\right) \\
& +\operatorname{Pf}\left(\begin{array}{ccccc}
(i, j)_{l} & \varphi_{i}^{(-2)}(l) & \varphi_{i}^{(-1)}(l) & \varphi_{i}^{(0)}(l) & \varphi_{i}^{(0)}(l+1) \\
& & 0 & 0 & b^{2} \\
& & & 0 & -b \\
& & & & 1
\end{array}\right) \\
& =\operatorname{Pf}\left(1,2, \cdots, 2 N, d_{-1}, d^{l}\right)_{l}+\operatorname{Pf}\left(1,2, \cdots, 2 N, d_{-2}, d_{-1}, d_{0}, d^{l}\right)_{l},
\end{aligned}
$$


Integrable semi-discretizations of the reduced Ostrovsky equation

thus, we get

$$
\frac{1}{3}\left(\left(\partial_{s}-b\right)^{3}-\left(\partial_{r}-b^{3}\right)\right) \tau_{l+1}=\operatorname{Pf}\left(1,2, \cdots, 2 N, d_{-2}, d_{-1}, d_{0}, d^{l}\right)_{l} .
$$

Then an algebraic identity of pfaffian [42]

$$
\begin{aligned}
& \operatorname{Pf}\left(\cdots, d_{-2}, d_{-1}, d_{0}, d^{l}\right) \operatorname{Pf}(\cdots)=\operatorname{Pf}\left(\cdots, d_{-2}, d_{-1}\right) \operatorname{Pf}\left(\cdots, d_{0}, d_{1}\right) \\
& \quad-\operatorname{Pf}\left(\cdots, d_{-2}, d_{0}\right) \operatorname{Pf}\left(\cdots, d_{-1}, d^{l}\right)+\operatorname{Pf}\left(\cdots, d_{-2}, d^{l}\right) \operatorname{Pf}\left(\cdots, d_{0}, d_{1}\right),
\end{aligned}
$$

derives

$\frac{1}{3}\left(\left(\partial_{s}-b\right)^{3}-\left(\partial_{r}-b^{3}\right)\right) \tau_{l+1} \times \tau_{l}=\frac{1}{3}\left(\partial_{s}^{3}-\partial_{r}\right) \tau_{l} \times \tau_{l+1}-\partial_{s}^{2} \tau_{l} \times\left(\partial_{s}-b\right) \tau_{l+1}+\left(\partial_{s}-b\right)^{2} \tau_{l+1} \times \partial_{s} \tau_{l}$,

which is equivalent to

$$
\left(\left(D_{s}-b\right)^{3}-\left(D_{r}-b^{3}\right)\right) \tau_{l+1} \cdot \tau_{l}=0 .
$$

Next, we preform a reduction in parallel to period 3 reduction for the continuous case. Imposing the same reduction condition (2.45), which is also of the form

$$
\frac{1}{p_{i}^{3}}+\frac{1}{p_{2 N+1-i}^{3}}=b^{2}\left(\frac{1}{p_{i}}+\frac{1}{p_{2 N+1-i}}\right),
$$

and note that $\tau_{l}$ is rewritten as

$$
\tau_{l}=\left(\prod_{i=1}^{2 N} \varphi_{i}^{(0)}(l)\right) \operatorname{Pf}\left(\frac{\delta_{j, 2 N+1-i} c_{i, j}}{\varphi_{i}^{(0)}(l) \varphi_{2 N+1-i}^{(0)}(l)}+\frac{p_{i}-p_{j}}{p_{i}+p_{j}}\right),
$$

it can easily shown that the pfaffian $\tau_{l}$ satisfies

$$
\partial_{r} \tau_{l}=b^{2} \partial_{s} \tau_{l}
$$

therefore we have

$$
\left(D_{s}^{3}-3 b D_{s}^{2}+2 b^{2} D_{s}\right) \tau_{l+1} \cdot \tau_{l}=0 .
$$

In what follows, we construct a semi-discrete reduced Ostrovsky equation based on Eq. (3.10). First, by using the following relations

$$
\begin{aligned}
& \frac{D_{s} \tau_{l+1} \cdot \tau_{l}}{\tau_{l+1} \tau_{l}}=\left(\ln \frac{\tau_{l+1}}{\tau_{l}}\right)_{s}, \\
& \frac{D_{s}^{2} \tau_{l+1} \cdot \tau_{l}}{\tau_{l+1} \tau_{l}}=\left(\ln \left(\tau_{l+1} \tau_{l}\right)\right)_{s s}+\left(\left(\ln \frac{\tau_{l+1}}{\tau_{l}}\right)_{s}\right)^{2}, \\
& \frac{D_{s}^{3} \tau_{l+1} \cdot \tau_{l}}{\tau_{l+1} \tau_{l}}=\left(\ln \frac{\tau_{l+1}}{\tau_{l}}\right)_{s s s}+3\left(\ln \frac{\tau_{l+1}}{\tau_{l}}\right)_{s}\left(\ln \left(\tau_{l+1} \tau_{l}\right)\right)_{s s}+\left(\left(\ln \frac{\tau_{l+1}}{\tau_{l}}\right)_{s}\right)^{3},
\end{aligned}
$$

one obtains

$$
\begin{aligned}
\left(\ln \frac{\tau_{l+1}}{\tau_{l}}\right)_{s s s}= & \left(b-\left(\ln \frac{\tau_{l+1}}{\tau_{l}}\right)_{s}\right) \\
& {\left[3\left(\ln \left(\tau_{l+1} \tau_{l}\right)\right)_{s s}-\left(\ln \frac{\tau_{l+1}}{\tau_{l}}\right)_{s}\left(2 b-\left(\ln \frac{\tau_{l+1}}{\tau_{l}}\right)_{s}\right)\right], }
\end{aligned}
$$


from Eq. (3.10). Next, by using the discrete hodograph transformation (2.30) and dependent variable transformation (2.31), Eq. (3.11) reads

$$
\frac{d}{d s}\left(u_{l+1}-u_{l}\right)=\frac{3}{2} \delta_{l}\left(u_{l}+u_{l+1}\right)-\frac{1}{4} \delta_{l}\left(\delta_{l}^{2}-4 b^{2}\right) .
$$

Obviously, the evolution equation for nonuniform mesh $\delta_{l}$ remains the same as Eq. (2.33). In summary

Theorem 3.2. The bilinear equation

$$
\left(\frac{1}{b} D_{s}^{3}-3 D_{s}^{2}+2 b D_{s}\right) \tau_{l+1} \cdot \tau_{l}=0
$$

determines a semi-discrete analogue of the reduced Ostrovsky equation (1.1)

$$
\left\{\begin{array}{l}
\frac{d}{d s}\left(u_{l+1}-u_{l}\right)=\frac{3}{2} \delta_{l}\left(u_{l}+u_{l+1}\right)-\frac{1}{4} \delta_{l}\left(\delta_{l}^{2}-4 b^{2}\right) \\
\frac{d \delta_{l}}{d s}=u_{l+1}-u_{l}
\end{array}\right.
$$

through the dependent variable transformation $u_{l}=-2\left(\ln \tau_{l}\right)_{s s}$ and the discrete hodograph transformation $x_{l}=2 l b-2\left(\ln \tau_{l}\right)_{s}, \delta_{l}=x_{l+1}-x_{l}$.

Now we turn to check if Eq. (3.13) converges to Eq. (1.1) in the continuous limit. By dividing $\delta_{l}$ on both sides of Eq. (3.12), we have

$$
\frac{1}{\delta_{l}} \frac{d}{d s}\left(u_{l+1}-u_{l}\right)=\frac{3}{2}\left(u_{l}+u_{l+1}\right)-\frac{1}{4} \delta_{l}^{2}+b^{2},
$$

which converges to exactly the reduced Ostrovsky equation (1.1)

$$
\partial_{x}\left(\partial_{t}+u \partial_{x}\right) u=3 u
$$

as $b \rightarrow 0\left(\delta_{l} \rightarrow 0\right)$.

Regarding the $N$-soliton solution, it is obvious that Eq. (3.13) admits the same solution as the semi-discrete reduced Ostrovsky equation (2.33) proposed previously.

So far, we have constructed semi-discrete analogues of the reduced Ostrovsky equation (1.1) and its differentiation form (1.3). In light of the link between (1.3) and (1.1), let us find a connection between (2.41) and (3.13). First, by taking a backward difference of Eq. (3.14), we obtain

$$
\frac{1}{\delta_{l}} \frac{d}{d s}\left(u_{l+1}-u_{l}\right)-\frac{1}{\delta_{l-1}} \frac{d}{d s}\left(u_{l}-u_{l-1}\right)=\frac{3}{2}\left(u_{l+1}-u_{l-1}\right)-\frac{1}{4}\left(\delta_{l}^{2}-\delta_{l-1}^{2}\right) .
$$

On the other hand, by substituting the third equation into the first equation in (2.41) and eliminating $m_{l}$, one arrives at exactly the same equation (3.15).

Remark 3.3. Although we have derived semi-discrete analogues of the reduced Ostrovsky equation (1.1) and its differentiation form (1.3) from totally different bilinear equations, the connection between them is clear here. In other words, the semi-discrete analogue for the short wave limit of the DP equation is simply a backward difference of semi-discrete reduced Ostrovsky equation. This finding corresponds to the fact that a differentiation of the reduced Ostrovsky equation (1.1) with respect to spatial variable $x$ gives rise to the short wave limit of the DP equation (1.3) in the continuous case. Forward difference and differentiation are 
two typical operators corresponding to discrete systems and continuous systems, respectively. In the world of integrable systems, we observe a perfect correspondence between these two operators and discrete and continuous systems.

Lastly, for the sake of convenience, we list the $\tau$-functions for one- and two-soliton solutions.

\section{One-soliton}

For $N=1$, we have

$\tau_{l}=\operatorname{Pf}(1,2)=c_{1}+\frac{p_{1}-p_{2}}{p_{1}+p_{2}} e^{\eta_{1}(l)+\eta_{2}(l)}$,

where $c_{1}$ is a nonzero constant,

$$
e^{\eta_{i}(l)}=\left(\frac{1+b p_{i}}{1-b p_{i}}\right)^{l} e^{p_{i}^{-1} s+\xi_{i 0}}
$$

and $p_{1}, p_{2}$ are related by a constraint

$$
\frac{1}{p_{1}^{3}}+\frac{1}{p_{2}^{3}}=b^{2}\left(\frac{1}{p_{1}}+\frac{1}{p_{2}}\right) \text {. }
$$

Two-soliton

For $N=2$, we have

$$
\begin{aligned}
& \tau_{l}=\operatorname{Pf}(1,2,3,4)=\operatorname{Pf}(1,2) \operatorname{Pf}(3,4)-\operatorname{Pf}(1,3) \operatorname{Pf}(2,4)+\operatorname{Pf}(1,4) \operatorname{Pf}(2,3) \\
& =\frac{p_{1}-p_{2}}{p_{1}+p_{2}} e^{\eta_{1}(l)+\eta_{2}(l)} \times \frac{p_{3}-p_{4}}{p_{3}+p_{4}} e^{\eta_{3}(l)+\eta_{4}(l)}-\frac{p_{1}-p_{3}}{p_{1}+p_{3}} e^{\eta_{1}(l)+\eta_{3}(l)} \times \frac{p_{2}-p_{4}}{p_{2}+p_{4}} e^{\eta_{2}(l)+\eta_{4}(l)} \\
& \quad+\left(c_{1}+\frac{p_{1}-p_{4}}{p_{1}+p_{4}} e^{\eta_{1}(l)+\eta_{4}(l)}\right)\left(c_{2}+\frac{p_{2}-p_{3}}{p_{2}+p_{3}} e^{\eta_{2}(l)+\eta_{3}(l)}\right),
\end{aligned}
$$

under the condition

$$
\frac{1}{p_{1}^{3}}+\frac{1}{p_{4}^{3}}=b^{2}\left(\frac{1}{p_{1}}+\frac{1}{p_{4}}\right), \quad \frac{1}{p_{2}^{3}}+\frac{1}{p_{3}^{3}}=b^{2}\left(\frac{1}{p_{2}}+\frac{1}{p_{3}}\right) .
$$

Letting $c_{1}=c_{2}=1$ and $e^{\gamma_{1}}=\frac{p_{1}-p_{4}}{p_{1}+p_{4}}$ and $e^{\gamma_{2}}=\frac{p_{2}-p_{3}}{p_{2}+p_{3}}$, the above $\tau$-function can be rewritten as $\tau_{l}=1+e^{\eta_{1}(l)+\eta_{4}(l)+\gamma_{1}}+e^{\eta_{2}(l)+\eta_{3}(l)+\gamma_{2}}+b_{12} e^{\eta_{1}(l)+\eta_{2}(l)+\eta_{3}(l)+\eta_{4}(l)+\gamma_{1}+\gamma_{2}}$,

where

$b_{12}=\frac{\left(p_{1}-p_{2}\right)\left(p_{1}-p_{3}\right)\left(p_{4}-p_{2}\right)\left(p_{4}-p_{3}\right)}{\left(p_{1}+p_{2}\right)\left(p_{1}+p_{3}\right)\left(p_{4}+p_{2}\right)\left(p_{4}+p_{3}\right)}$.

In the continuous limit $b \rightarrow 0$, it is obvious that above one- and two-soliton solutions for semi-discrete reduced Ostrovsky equation converge to the one- and two-soliton solutions for the reduced Ostrovsky equation listed in [28]. 


\section{Conclusion and further topics}

There are two versions of the reduced Ostrovsky equation, one is the original form (1.1), the other is its differentiation form, or is also called the short wave limit of the DP equation (1.3). In the present paper, we have constructed their integrable semi-discretizations separately based on their different bilinear forms. Two versions of integrable semi-discretizations of the reduced Ostrovsky equation share the same $N$-soliton solution in terms of pfaffians, which converges to the $N$-soliton solution of the continuous Ostrovsky equation (1.1), as well as its differentiation form (1.3). The connection between two versions of integrable discretizations is made clear. In the continuous case, the short wave limit of the DP equation (1.3) is the differentiation form of the reduced Ostrovsky equation, whereas in the discrete case, the semidiscrete short wave limit of the DP equation is the forward difference for the semi-discrete reduced Ostrovsky equation.

Similar to our previous results [43, 44, 45], the semi-discrete reduced Ostrovsky equation proposed here can be served as an integrable numerical scheme, the so-called self-adaptive moving mesh method, for the numerical simulation. It seems that the semi-discrete reduced

Ostrovsky equation (3.13) has more advantages than the semi-discrete analogue of the short wave limit of the DP equation in serving as a self-adaptive moving mesh method. We would like to report our results in this aspect in a forthcoming paper. Finally, we haven't succeeded in constructing an integrable fully discrete reduced Ostrovsky equation. If we could have done so, then a newly integrable discrete Tzitzeica equation might be constructed due to a direct link between these two equations. It is a further topic to be explored in the future. Another problem to be solved is the integrable discretization of the DP equation which is a more challenging problem in compared with the ones of the Camassa-Holm equation and the reduced Ostrovsky equation. We are tacking this problem based on our previous work on the DP equation [46].

\section{Acknowledgment}

This work of BF is partially supported by the National Natural Science Foundation of China (No. 11428102).

\section{References}

[1] Ostrovsky L A 1978 Oceanology 18, 119-125

[2] Stepanyants Y A 2006 Chaos, Solitons \& Fractals 28, 193-204

[3] Parkes E J 2007 Chaos, Solitons \& Fractals 31, 602-610

[4] Hunter J 1990 Lectures in Appl. Math. 26, 301-316

[5] Vakhnenko V O 1992 J. Phys. A: Math. Gen. 25, 4181-4187

[6] Vakhnenko V O 1999 J. Math. Phys. 40, 2011-20

[7] Vakhnenko V O and Parkes E J 1998 Nonlinearity 11, 1457-1464

[8] Morrison A J, Vakhnenko V O and Parkes E J 1999 Nonlinearity, 12, 1427-1437

[9] Vakhnenko V O and Parkes E J 2002 Chaos, Solitons \& Fractals 13, 1819-1826

[10] Liu Y, Pelinovsky D and Sakovich A 2010 SIAM J. Math. Anal. 42, 1967-1985 
[11] Brunelli J C and Sakovich S 2013 Commun. Nonlinear Sci. Numer. Simul. 18, 5662

[12] Boutet de Monvel A and Shepelsky D 2015 J. Phys. A 48, 035204

[13] Boyd J P 2005 Euro. Jnl. of Appl. Math. 16, 65-81

[14] Hone A N W and Wang J P 2003 Inverse Problems 19, 129-145

[15] Matsuno Y 2006 Phys. Lett. A 359, 451-457

[16] Degasperis A and Procesi M 1999 Asymptotic integrability, in Symmetry and Perturbation Theory, edited by A. Degasperis and G. Gaeta, World Scientific 23-37

[17] Matsuno Y 2005 Inverse Problems 21, 1553-1570

[18] Matsuno Y 2005 Inverse Problems 21, 2085-2101

[19] Grimshaw R H J, Helfrich K and Johnson E R 2012 Stud. Appl. Math. 129, 41436

[20] Kraenkel R A, Leblond H and Manna M A 2014 J. Phys. A 47, 025208

[21] Tzitzeica G 1910 C. R. Acad. Sci. 150, 9556

[22] Tzitzeica G 1910 C. R. Acad. Sci. 150, 12279

[23] Willox R 2005 Glasgow Math. J. 47A, 22131

[24] Nimmo J J C and Ruijsenaars S N M 2009 J. Math. Phys. 50, 043511

[25] Dodd R K and Bullough R K 1977 Proc. R. Soc. A 352, 481503

[26] Mikhailov A V 1979 JETP Lett. 30, 4148

[27] Mikhailov A V 1981 Physica D 3, 73117

[28] Feng B.-F., Maruno K and Ohta Y 2012 J. Phys. A 45, 355203

[29] Ablowitz M J and Ladik J 1975 J. Math. Phys. 16, 598

[30] Ablowitz M J and Ladik J 1976 Stud. Appl. Math. 55, 213

[31] Hirota R 1977 J. Phys. Soc. Jpn. 43, 1424

[32] Hirota R 1977 J. Phys. Soc. Jpn. 43, 2079

[33] Hirota R 1977 J. Phys. Soc. Jpn. 43, 2074

[34] Date E, Jimbo M and Miwa T 1982 J. Phys. Soc. Jpn. 51, 4116-4127

[35] Date E, Jimbo M and Miwa T 1982 J. Phys. Soc. Jpn. 51, 4125-4131

[36] Date E, Jimbo M and Miwa T 1983 J. Phys. Soc. Jpn. 52, 388-393

[37] Date E, Jimbo M and Miwa T 1983 J. Phys. Soc. Jpn. 52, 761-765

[38] Date E, Jimbo M and Miwa T 1983 J. Phys. Soc. Jpn. 52, 766-771

[39] Jimbo M and Miwa T 1983 Publ. RIMS. Kyoto Univ. 19, 943-1001

[40] Hirota R 1981 J. Phys. Soc. Jpn. 50, 3785-3791

[41] Suris Y B 2003 The Problem of Integrable Discretization: Hamiltonian Approach, Birkhäuser.

[42] Hirota R 2004 The Direct Method in Soliton Theory, Cambridge University Press.

[43] Ohta Y, Maruno K and Feng B-F 2008 J. Phys. A 41, 355205

[44] Feng B-F, Maruno K and Ohta Y 2010 J. Comput. Appl. Math 235, 229-243

[45] Feng B F, Maruno K and Ohta Y 2010 J. Phys. A 43, 085203

[46] Feng B-F, Maruno K and Ohta Y 2013 J. Phys. A 46, 045205 